\title{
Estudio de la Contaminación por Metales Ecotóxicos en Sedimentos en la Bahía de Ite, Tacna
}

\section{Study of Pollution by Metals Ecotoxic in Sediments in the Bay of Ite, Tacna}

\author{
${ }^{1}$ Lorenzo Walter Ibárcena Fernández
}

\begin{abstract}
RESUMEN:
La costa peruana se han visto sometida a una fuerte presión por contaminación humana e industrial, especialmente en las ziltimas cuatro décadas, lo que ha afectado seriamente a la integridad del ecosistema marino. En el presente trabajo se hace um estudio de los niveles de contaminación por elementos ecotóxicos en sedimentos marinos por efectos antropogénicos, de los siguiente elementos: $\mathrm{Cu}, \mathrm{Zn} . \mathrm{Cd}, \mathrm{As}, \mathrm{Hg}, \mathrm{Ph}$ y Fe, en la Bahia de Ite. Distrito de Ite. Provincia de Jorge Basadre Grohmann de Tacna, determinando la incidencia que tendrian los mismos sobre la fauna bentónica de la zona, como consecuencia del vertimiento por más de 35 años de los relaves mineros provenientes de las minas de Toquepala y Cuajone. Los resultados obtenidos de los metales ecotóxicos $\mathrm{Cu}, \mathrm{Zn} . \mathrm{As}, \mathrm{Cd}, \mathrm{Hg}, \mathrm{Pb}, \mathrm{Fe}$, analizados que se encuentran en los sedimentos superficiales de la Bahía de Ite, en orden decreciente son: $\mathrm{Fe}>\mathrm{C} u>\mathrm{Zn}>\mathrm{As}>\mathrm{Pb}>\mathrm{Cd}>\mathrm{Hg}$. Los valores promedios reportados son los siguientes: $C u=608.063 \mathrm{mg} / \mathrm{kg}, \mathrm{Zn}=39.923 \mathrm{mg} / \mathrm{kg}, \mathrm{As}=8.66$ $\mathrm{mg} / \mathrm{kg} . \mathrm{Cd}=0.41 \mathrm{mg} / \mathrm{kg}, \mathrm{Hg}<0.01 \mathrm{mg} / \mathrm{kg}, P b=8.472 \mathrm{mg} / \mathrm{kg} . \mathrm{Fe}=33078.63 \mathrm{mg} / \mathrm{kg}$. Estos resultados fueron comparados con análisis en sedimentos marinos superficiales efectuados en la zona sur. en el lugar denominado Las Mesas o Punta Mesa, área que consideramos libre de efectos contaminantes antropogénicos. De igual manera se realizo una verificación en la zona norte. Santa Rosa para poder establecer si en la zona existe incidencia de los efectos contaninantes de los relaves mineros depositados en la Batiade lie.
\end{abstract}

Palabras clave: contaminación. ecotóxicos, metales pesados, sedimentos marinos.

\section{ABSTRACT:}

The Perwian coast has heen submiled to heavy pressure firom human and industrial pollution, especially in the last four decades, which has seriously affected the marine ecosystem integrity. This paper presents a study of pollution levels in marine sediments by ecotoxic elements originated by anthropogenic effects, in the following elements: Cu, Zn, Cd, As, Hg. Ph and Fe; in the Bay of Ite, District of Ite, . Forge Basadre Grohmann province of Tacna, determining the incidence they would have on the benthic fanma of the area, as a conse'quence of the dumping for more than 35 vears of failings from Toquepala and Cuajone. The results of the analysed ecotoxic metats $\mathrm{C} u, \mathrm{Zn}, \mathrm{As}$. Cd, Hg, Pb, Fe, found in sediments surface in the Bay of lte, in descending order are: $\mathrm{Fe}>\mathrm{Cu}>\mathrm{Zn}>\mathrm{As}>\mathrm{Pb}>\mathrm{Cd}>\mathrm{Hg}$. The averuge values reported are the following: $\mathrm{Cu}=$ $608.063 \mathrm{mg} / \mathrm{kg}, Z \mathrm{n}=39.923 \mathrm{mg} / \mathrm{kg}, \mathrm{As}=8.66 \mathrm{mg} \mathrm{kg} . \mathrm{Cd}=0.11 \mathrm{mg} / \mathrm{kg} . \mathrm{Hg}<0.01 \mathrm{mg} / \mathrm{kg}, \mathrm{Pb}=8.472 \mathrm{mg} / \mathrm{kg}$, $\mathrm{Fe}=33078.63 \mathrm{mg} / \mathrm{kg}$. These results were compared with analys is performed in superficial marine sediments in the south, at a place called Las Mesas or Punta Mesa. cin area considered free of anthropogenic pollutants effects. In the same way, a check in the north. on a place called Santa Rosa was made to establish if there was incidence of the polluting effeets of mining wastes stored in the Bav of Ite.

Keywords: pollution, ecotoxic, heavy metals, marine sediments

\footnotetext{
'Doctor en Ciencias y Tecnologias Medioambientales, Maestro en Ciencias con Mencion en Computacion e informática. Ingeniero Pesquero. Facultad de Ciencias Agropecuarias. Universidad Nacional Jorge Basadre Cirohmann
} 


\section{INTRODUCCIÓN}

Para el Perú, con sus 3080 kilómetros de litoral, la zona costera reviste una singular importancia, ya que además de los recursos energéticos y minerales que posee, representa también una fuente significativa de de alimentos. Sin embargo, en la actualidad las bahías, estuarios y lagunas costeras del país encaran serios problemas de contaminación, los cuales producen daños considerables a los organismos que los habitan.

Ellos pueden dar lugar a fenómenos de toxicidad, aún en concentraciones muy bajas, lo que afectaria al desarrollo y vida de los organismos y consecuentemente a la cadena alimentaria. Generalmente, los metales transportados a los ecosistemas costeros se fijan fácilmente sobre el material particulado, lo que hace que su destino principal sean los sedimentos costeros. Por ello, los sedimentos son un almacén altamente concentrado de metales, con concentraciones superiores a las aguas adyacentes. Una vez que los metales son incorporados a los sedimentos costeros, el cambio en las condiciones redox del medio y los procesos de redistribución de los metales en las distintas fases del sedimento afectan a su biodisponibilidad y por lo tanto, a la calidad ambiental del sistema, de tal manera, la medición de la concentración de cada especie química en los sedimentos superficiales proporciona información sobre su comportamiento geoquimico, biodisponibilidad $\mathrm{y} / \mathrm{o}$ toxicidad e indica si la presencia de metales pesados, en el ambiente, es una contribución natural procedente de la corteza terrestre o existen aportes contaminantes generados por las actividades humanas (Lewis y Landing 1992; García -Montelongo et al., 1994; Balls et al., 1997; Ponce et al., 2000; Williamson y Morrisey 2000; Codina y Pérez 2001; Martínez y Senior 2002; Durán et al., 2004).

\section{MARCO METODOLÓGICO}

\subsection{Ubicación de la zona de estudio:}

El área de interés en la cual se considero efectuar el estudio de los niveles de metales pesados en sedimentos superficiales está ubicada en el Distrito de Ite, Provincia de Jorge Basadre Grohmann de Tacna, La Bahia de Ite, por el norte el área de estudio alcanzará hasta la zona de Punta Alfarillo, y por el sur hasta Meca Grande, en la provincia de Jorge Basadre, las zonas de control se encuentran al sur en la zona de Punta Mesa, y al norte en Santa Rosa, las que se muestran en la Figura $\mathrm{N}^{\circ} 01$

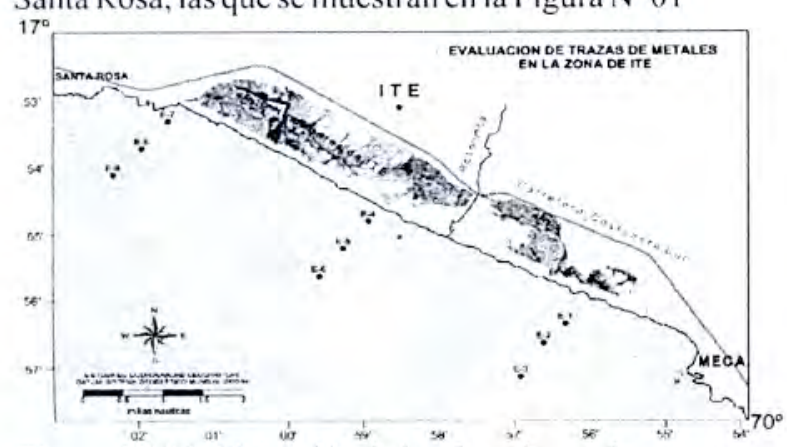

Figura $\mathrm{N}^{\circ} 01$ : Ubicación de las Estaciones de muestreo, Bahía de Ite

\subsection{Bahía de Ite}

Se ubica al Sur del Perú en la Región Tacna, Provincia de Jorge Basadre, Distrito de Ite, lugar denominado Playa Inglesa (Figura 01), formada por una playa artificial de relaves mineros, la zona cuenta con vegetación, formando los denominados humedales de Ite, no existen en la zona descargas antropogenicas de origen domestico. El agua que alimenta los humedales proviene de las filtraciones de la represa de Quebrada Honda, donde se almacenan en la actualidad los relaves mineros que generan las minas de Cuajone y Toquepala.

\subsubsection{Muestreo de sedimentos}

El presente estudio tiene por finalidad muestrear la zona indicada para la cuantifícación de los niveles de los siguientes metales ecotóxicos: cobre, arsénico, zinc, cadmio, mercurio, plomo y hierro.

El manejo y preservación de las muestras en el muestreo se efectuó con referencia a la Normatividad de la Agencia de Protección Ambiental (U.S. Environmental Protection Agency, 2004), Método de SW-846. Material y equipo de muestreo son frascos o bolsas de polietileno o polipropileno, draga, hielo y cubetas.

Se tomaran 3 replicas por estación para seleccionar los sedimentos haciendo uso de una draga tipo Van Veen de $0.06 \mathrm{~m} 2$. Una de las replicas se utilizo para obtener una sub muestra de aproximadamente $800 \mathrm{~g}$, para los análisis respectivos de materia orgánica, $\mathrm{pH}$ y metales pesados en sedimento. Las muestras, se colocaron en bolsas plásticas impermeables debidamente codificadas, proporcionadas por el laboratorio en el cual se realizo los análisis. Seguidamente se colocaron en un cooler donde se refrigeraron, hasta su llegada al laboratorio.

\subsubsection{Análisis de metales pesados en sedimentos}

Los equipos y aparatos utilizados en el laboratorio son generalmente fuentes de contaminación de las muestras objeto de análisis, por dicho motivo para realizar este análisis se eligió un laboratorio certificado, denominado Inspectorate Services Perú S.A.C. de la capital de la República.

\section{RESULTADOS}

\subsection{Resultados Metales Ecotóxicos Bahía de Ite}

Resultados de los análisis en sedimentos marinos efectuados en la Bahia de Ite, realizados en el laboratorio acreditado Inspectorate Services Perú SAC, de la capital de la República, presentamos en este capitulo.

Cobre: Las concentraciones de cobre en sedimentos marinos de la Bahía de Ite, se muestran en la Tabla 2, se puede observar que las concentraciones más bajas se encuentran en las E-9 con $288.43 \mathrm{mg} / \mathrm{kg}$, seguida de la E-6 con 469.14 y la E-3 con $505.28 \mathrm{mg} / \mathrm{kg}$. Encontrándose las concentraciones más altas en la E-4 
con $935.14 \mathrm{mg} / \mathrm{kg}$ y la segunda más alta la E-1 con $845.67 \mathrm{mg} / \mathrm{kg}$.

Los valores de cobre aumentan considerablemente conforme las estaciones de muestreo se alejan de la costa, lo que nos indica que los niveles elevados de cobre total encontrados son debido a los relaves mineros almacenados en la zona provenientes de las minas de Toquepala y Cuajone

En la Figura 2a se muestra la distribución gráfica de las concentraciones del cobre como contaminante en sedimentos marinos a través de isolineas, el color rojo muestra la concentración mayor de dicho metal, los colores claros indican la reducción paulatina, conforme se alejan de la costa.

Zinc: Las concentraciones de Zinc en sedimentos marinos de la Bahía de Ite, se muestran en la Tabla 1, se puede observar que las concentraciones más bajas se encuentran en las E-9 con $28.50 \mathrm{mg} / \mathrm{kg}$, seguida de la E8 con 33.97. Registrándose las concentraciones más altas en la E-2 con $44.63 \mathrm{mg} / \mathrm{kg}$ y la segunda más alta la E-5 con $41.63 \mathrm{mg} / \mathrm{kg}$.

Arsénico: Las concentraciones de Arsénico en sedimentos marinos de la Bahía de Ite, se inuestran en la Tabla 1, se puede notar que las concentraciones más bajas se encuentran en las E-7 con $6.01 \mathrm{mg} / \mathrm{kg}$, seguida de la E-2 con $6.73 \mathrm{mg} / \mathrm{kg}$. Resultando las concentraciones más altas en la E-6 con $11.04 \mathrm{mg} / \mathrm{kg}$ y la segunda de la E-9 con $10.63 \mathrm{mg} / \mathrm{kg}$.

Cadmin: Las concentraciones de cadmio en sedimentos marinos de la Bahía de Ite, se presentan en la Tabla 1, se puede observar que las concentraciones más bajas se registran en las E-5 con $0.22 \mathrm{mg} / \mathrm{kg}$, seguida de la E-7 con $0.27 \mathrm{mg} / \mathrm{kg}$. Reportándose las concentraciones más altas en la E-6 con $0.72 \mathrm{mg} / \mathrm{kg}$. seguida de la E-9 con $0.68 \mathrm{mg} / \mathrm{kg}$.

Los valores de cadmio aumentan conforme las estaciones de muestreo se alejan de la costa, Figura $2 \mathrm{~d}$ el moviniento de las concentraciones a zonas alejadas se debe a los movimientos generados por las corrientes marinas y a la dispersión de los sedimentos.
Mercurio: Las concentraciones de mercurio reportados en los análisis de laboratorio, no superaron los niveles mínimos de cuantificación del equipo, lo que significa que la zona está libre de este metal pesado, según se muestra en la tabla 1.

Plomo: Las concentraciones de Plomo en sedimentos marinos de la Bahia de Ite, se presentan en la Tabla I, puede observarse que los valores menores se encuentran en las E-I con $6.18 \mathrm{mg} / \mathrm{kg}$, seguida de la E-7 con 7.45 $\mathrm{mg} / \mathrm{kg}$. Encontrándose las concentraciones más altas en la E-9 con $10.50 \mathrm{mg} / \mathrm{kg}$ y la E-3 con $9.65 \mathrm{mg} / \mathrm{kg}$.

Hiero: Las concentraciones de hierro en sedimentos marinos de la Bahía de Ite, se presenta en la Tabla 1, se puede observar que las concentraciones más bajas se encuentran en las E-7 con $14596.37 \mathrm{mg} / \mathrm{kg}$, seguida de la E-9 con $22175.96 \mathrm{mg} / \mathrm{kg}$. Las concentraciones mayores fueron reportadas en la $\mathrm{E}-4$ con $81106.98 \mathrm{mg} / \mathrm{kg}$ segunda de la E-6 con $47230.51 \mathrm{mg} / \mathrm{kg}$.

\subsection{Resultados Metales Ecotóxicos en Punta Mesa y Santa Rosa}

Para efectuar la comparación de los resultados de metales ecotóxicos en sedimentos marinos obtenidos en la Bahia de Ite, y la Zona de Punta Mesa y Santa Rosa se tomaron los datos del estudio de investigación realizado el año 2007 denominado: ESTUDIO DE LA CONTAMINACIÓN POR METALES PESADOS EN LASCOSTAS DETACNA.

\section{DISCUSIÓN}

En base a los resultados obtenidos se ha desarrollado la discusión que se presenta en el presente capítulo. Los metales trazas en los ecosistemas naturales oceánicos, marinos constituyen uno de los aspectos más importantes de la polución, ya que son la representación de la materia inorgánica antrópica, la cual es considerada como esencial y tóxica dependiendo tanto de la especiación quimica del elemento traza como su contenido (Bonilla et al., 1995; Aranda 1999).

La distribución horizontal y por estaciones de los metales traza $\mathrm{Cu}, \mathrm{Zn}, \mathrm{As}, \mathrm{Cd}, \mathrm{Hg}, \mathrm{Pb}$, Fe, se muestran en

Tabla $N^{\circ} 1$. Resultados de los Análisis en Sedimentos Marinos Bahía de Ite

\begin{tabular}{cccccccccc}
\hline $\begin{array}{c}\text { Estación } \\
\text { Muestreo }\end{array}$ & Fecha & $\begin{array}{c}\text { Profun } \\
\text { didad } \\
(\mathbf{m})\end{array}$ & $\begin{array}{c}\mathbf{C u} \\
(\mathbf{m g} / \mathbf{k g})\end{array}$ & $\begin{array}{c}\mathbf{Z n} \\
(\mathbf{m g} / \mathbf{k g})\end{array}$ & $\begin{array}{c}\mathbf{A s} \\
(\mathbf{m g} / \mathbf{k g})\end{array}$ & $\begin{array}{c}\mathbf{C d} \\
(\mathbf{m g} / \mathbf{k g})\end{array}$ & $\begin{array}{c}\mathbf{H g} \\
(\mathbf{m g} / \mathbf{k g})\end{array}$ & $\begin{array}{c}\mathbf{P b} \\
(\mathbf{m g} / \mathbf{k g})\end{array}$ & $\begin{array}{c}\mathbf{F e} \\
(\mathbf{m g} / \mathbf{K g})\end{array}$ \\
\hline E-1 & 20.07 .10 & 11.9 & 845.67 & 39.73 & 7.06 & 0.36 & $<0.01$ & 6.18 & 26532.99 \\
E-2 & 20.07 .10 & 17.8 & 652.73 & 44.63 & 6.73 & 0.29 & $<0.01$ & 7.49 & 25552.25 \\
E-3 & 20.07 .10 & 21.9 & 505.28 & 34.64 & 8.78 & 0.47 & $<0.01$ & 9.65 & 29927.27 \\
E-4 & 20.07 .10 & 10.2 & 935.14 & 40.94 & 10.46 & 0.28 & $<0.01$ & 9.27 & 81106.98 \\
E-5 & 20.07 .10 & 15.1 & 577.63 & 41.63 & 7.17 & 0.22 & $<0.01$ & 8.67 & 26153.67 \\
E-6 & 20.07 .10 & 17.7 & 469.14 & 33.98 & 11.04 & 0.72 & $<0.01$ & 8.44 & 47230.51 \\
E-7 & 17.07 .10 & 11.0 & 673.32 & 34.29 & 6.01 & 0.24 & $<0.01$ & 7.45 & 14596.37 \\
E-8 & 17.07 .10 & 15.9 & 525.23 & 33.97 & 10.06 & 0.43 & $<0.01$ & 8.60 & 24431.70 \\
E-9 & 17.07 .10 & 19.7 & 288.43 & 28.50 & 10.63 & 0.68 & $<0.01$ & 10.50 & 22175.96 \\
& Media & & 608.063 & 39.923 & 8.660 & 0.410 & 0.01 & 8.472 & 33078.63 \\
\multicolumn{2}{r}{ Desviación estándar } & 196.694 & 5.075 & 1.946 & 0.184 & 0.00 & 1.297 & 19998.77 \\
\multicolumn{2}{l}{ Límite Cuantificación laboratorio } & 0.01 & 0.02 & 0.04 & 0.02 & 0.01 & 0.02 & 0.31 \\
\hline
\end{tabular}


(a)

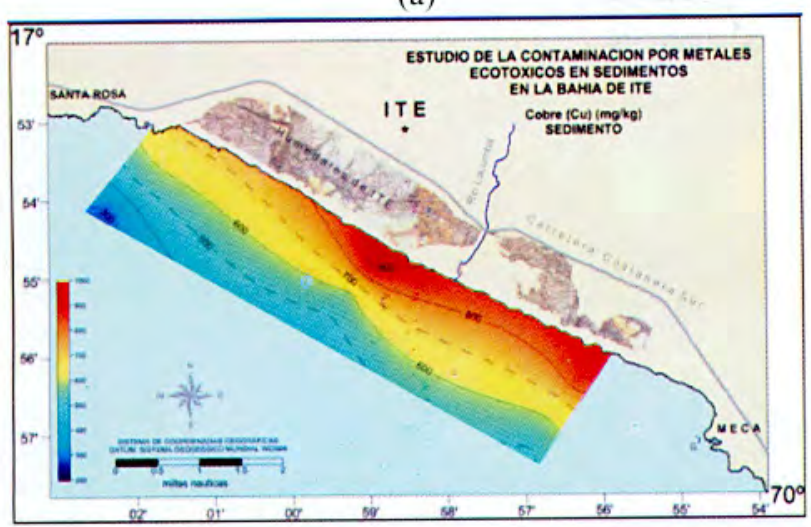

(c)

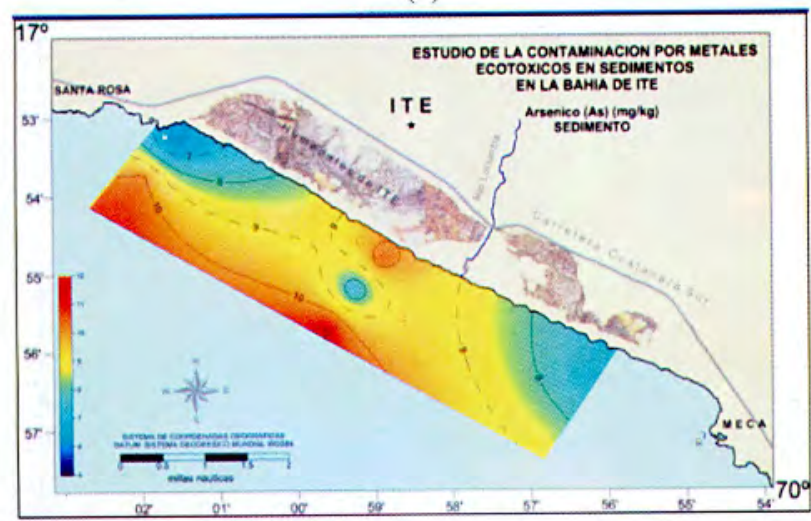

(e)

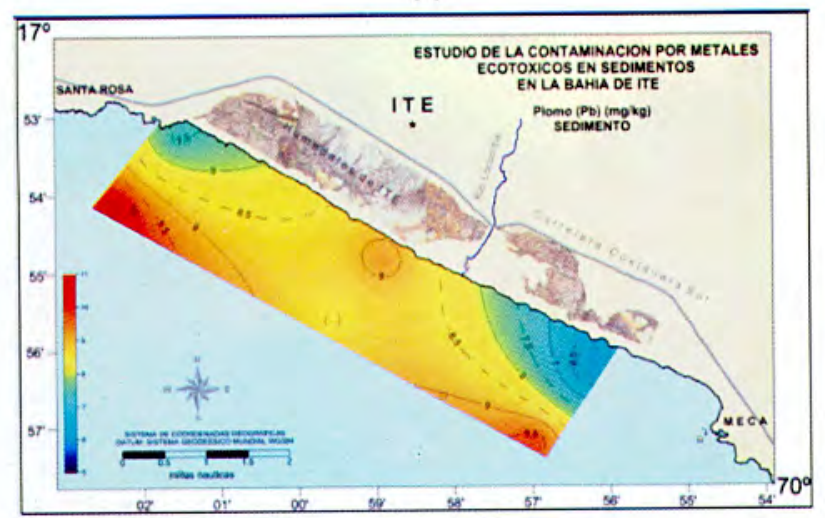

(b)

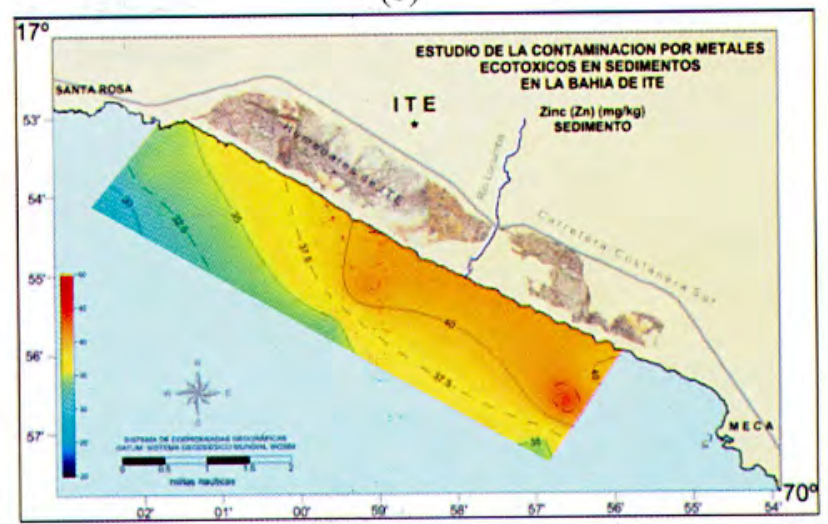

(d)

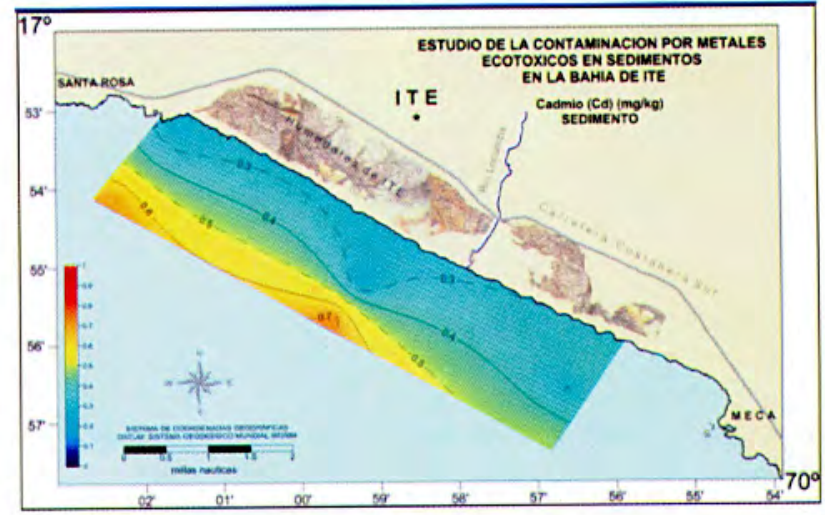

(f)

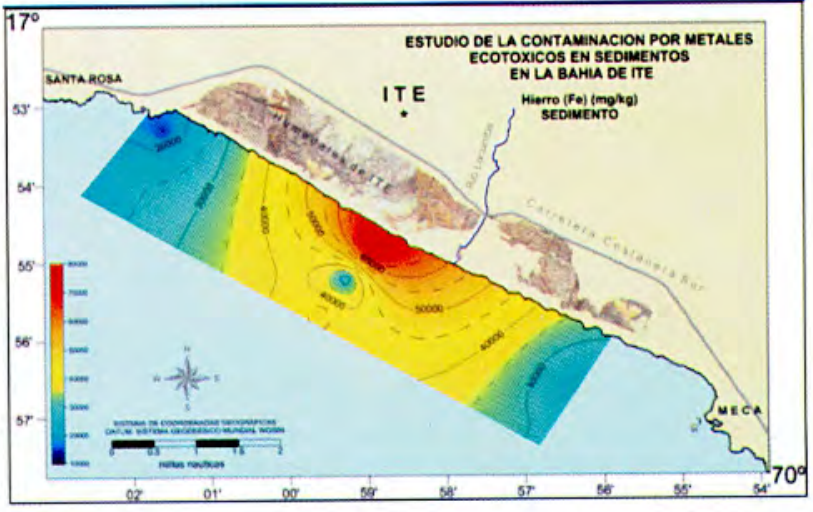

Figura $\mathbf{N}^{\mathbf{0}}$ 2: Isolineas de distribución de la contaminación por cobre, zinc, arsénico, cadmio, plomo y hierro en sedimentos marinos en la Bahía de Ite

las Figuras 2 y Tabla 1. De acuerdo al nivel de concentración los metales ecotóxicos que se encuentran en los sedimentos superficiales de la Bahía de Ite, en orden decreciente son: $\mathrm{Fe}>\mathrm{Cu}>\mathrm{Zn}>\mathrm{As}>\mathrm{Pb}>\mathrm{Cd}>$ $\mathrm{Hg}$. La reactividad y toxicidad de esos elementos, así como su impacto en la vida acuática varía de acuerdo a la concentración de cada metal.

El análisis de varianza realizado para comparar las zonas de estudio de la Bahía de Ite y Las Mesas, nos arroja diferencias significativas en las concentraciones obtenidas, en todos los metales ecotóxicos: $\mathrm{Cu}, \mathrm{Zn}, \mathrm{As}$, $\mathrm{Cd}, \mathrm{Pb}$ y Fe, a excepción del mercurio, de ambas aéreas de estudio.

Este resultado nos indica que la zona de comparación, Las Mesas ubicada al sur de la Bahía de Ite, nos permite establecer que la zona de estudio tiene niveles de metales ecotóxicos diferentes a las áreas donde no hay intervención de los relaves mineros.

Tabla $\mathbf{N}^{\circ}$ 2. Análisis de varianza de una vía para los parámetros medidos en sedimentos marinos de la Bahía de Ite y Las Mesas.

\subsection{Cobre}

La mayor concentración de cobre total $(935.14 \mathrm{mg} / \mathrm{kg})$ se detectó en la estación 4 (perfil centro, cerca de la 
costa) a una profundidad de $10.2 \mathrm{~m}$, esta concentración es muy superior al rango (50 a $68 \mu \mathrm{g} / \mathrm{g})$ del sedimento superficial de la bahía San Vicente, Chile calificada de no contaminada (Ahumada, 1992). El menor valor $(288.43 \mathrm{mg} / \mathrm{kg}$ ) se registró en la estación 9 a $2.7 \mathrm{~km}$ de la costa y a $19.7 \mathrm{~m}$ de profundidad. En la presente evaluación la distribución espacial de cobre es creciente hacia la boca del río Locumba; registrándose que la concentración de cobre total fue alta en áreas de influencia de las descargas provenientes de la actividad minero-metalúrgica.

Podemos indicar que, según los estándares establecidos para sedimentos por la Agencia de Protección Ambiental de Estados Unidos (USEPA), para el metal pesado cobre las concentraciones encontradas en la Bahía de Ite, están en la categoría de sedimentos con contaminación alta debido a que superan los $50 \mu \mathrm{g} / \mathrm{g}$. Se hace notar que todas las estaciones de muestreo superaron los niveles antes indicados para este elemento, lo que nos indicaria que toda la zona está en la categoria de alta contaminación según la USEPA (1991).

Todas las estaciones de muestreo están dentro del rango de sedimentos contaminados para cobre según la OME de Canadá, que establece como límite $25 \mu \mathrm{g} / \mathrm{g}$, para este metal, según los resultados obtenidos la Bahía de Ite se encuentra muy por arriba de este límite. Además de estar sobre el rango de causa de efectos tóxicos, establecidos por la NOAA, que establece como umbral $270 \mu \mathrm{g} / \mathrm{g}$, todas las estaciones de muestreo se encuentran sobre este nivel de referencia, solo la E-9 se encuentra cercana al limite de alarma con $288.43 \mathrm{mg} / \mathrm{kg}$.

La contaminación por metales pesados, como cobre, puede afectar la fotosintesis y el desarrollo de las algas, asi como las primeras etapas del desarrollo de los animales marinos (huevos, larvas, etc.) pudiendo causar la muerte de los mismos (UNESCO 1976; Aranda 1999).

De lo indicado se puede inferir que el impacto físico de los relaves (ocupación del litoral y los fondos marinos) ha provocado la desaparición de la flora y fauna típica que soporta el ecosistema marino impactado. Sin embargo la dimensión del daño generado por la dispersión del material de relaves en litoral y fondos marinos, derivado fundamentalmente por fenómenos físicos de afloramientos y por las corrientes marinas continuará por mucho tiempo.

\subsection{Zinc}

En base a los resultados de las concentraciones del metal pesado zinc obtenidas en la Bahia de Ite, se muestran en la Tabla 1 y Figura $2 b$, que presenta la distribución horizontal, mediante isolineas, del contenido de zinc en los sedimentos superficiales del ecosistema de la Bahía de Ite. Los valores reportados oscilan entre $28.50 \mathrm{mg} / \mathrm{kg}$ registrado en la E-9, a una profundidad de $19.7 \mathrm{~m}$ (a 7.92 $\mathrm{km}$ de la boca del río Locumba), y $44.63 \mathrm{mg} / \mathrm{kg}$ detectado en la E-2 a una profundidad de $17.8 \mathrm{~m}$ (a 3.73 $\mathrm{km}$ de la boca del río Locumba), con una media de
$36.923 \mathrm{mg} / \mathrm{kg}$, la cual está muy por debajo del valor de $110.0 \mu \mathrm{g} / \mathrm{g}$ de zinc para sedimentos no contaminados (Sadiq, 1992), lo que indica que la Bahía de Ite no está contaminada por zinc, es decir la causa de la no existencia de flora y fauna en la zona no se debería a la contaminación por zinc, puede deberse a la contaminación por otros elementos ecotóxicos. Si su concentración excediera la de $110 \mu \mathrm{g} / \mathrm{g}$, se podría inferir la probable degradación de la zona por efectos de la contaminación por zinc.

También podemos observar que, según los estándares para sedimentos marinos establecidos por la Agencia de Protección Ambiental de Estados Unidos (USEPA), para el metal pesado zinc las concentraciones encontradas en la Bahia de Ite, están en la categoría de sedimentos no contaminados debido a que no superan los $90 \mu \mathrm{g} / \mathrm{g}$. Se hace notar que todas las estaciones de muestreo no superaron los niveles antes indicados para este elemento, lo que nos indicaría que toda la zona está en la categoría de no contaminada según la USEPA (1991).

Todas las estaciones de muestreo están dentro del rango de sedimentos no contaminados para zinc según la OME de Canadá, que establece como limite $100 \mu \mathrm{g} / \mathrm{g}$, para este metal, según los resultados obtenidos la Bahía de Ite se encuentra muy por debajo de este límite. Además de estar bajo el rango de causa de efectos tóxicos, establecidos por la NOAA, que establece como umbral $150 \mu \mathrm{g} / \mathrm{g}$, con probabilidad baja que un elemento pueda producir efecto tóxico.

Efectuada la comparación entre los resultados de las concentraciones de zinc obtenidos en la Bahia de Ite, con una media de $36.923 \mathrm{mg} / \mathrm{kg}$, y los resultados que se obtuvo en la zona de Las Mesas con $12.38 \mathrm{mg} / \mathrm{kg}$, notamos que existe diferencia significativa entre los dos niveles de contaminación, y los valores medios encontrados en la zona norte de Santa Rosa con 10.86 $\mathrm{mg} / \mathrm{kg}$ (Tabla 2), significan que la proyección de la contaminación no tiene influencia significativa en la zona. El nivel promedio de zinc encontrado en sedimentos superficiales en Las Mesas fue de 12.38 $\mathrm{mg} / \mathrm{kg}$, valores bajos de este metal podrian tener un origen litogénico al igual que el hierro y el manganeso (Martínez G. 2002).

\subsection{Arsénico}

Los niveles de arsénico en los sedimentos superficiales del área de estudio fueron mixtos, fluctuando entre 6.01 y $11.04 \mathrm{mg} / \mathrm{kg}$, en las estaciones de muestreo (E-7) y (E6), respectivamente. Con un promedio de $8.66 \mathrm{mg} / \mathrm{kg}$, Tabla 1. Los resultados de las concentraciones del arsénico obtenidas en la Bahía de Ite, se muestran en la Tabla 1, además, en la Figura $2 \mathrm{c}$, podemos visualizar la distribución horizontal, mediante isolíneas, del contenido de arsénico en los sedimentos superficiales del ecosistema de la Bahia de Ite.

Respecto de estándares internacionales, el criterio de calidad de sedimentos marinos para el arsénico total, establecidos por la Agencia de Protección Ambiental de 
Estados Unidos (USEPA), registrados en la Bahía de Ite, están en la categoría de sedimentos con contaminación moderada a alta, debido a que superan los $8.0 \mu \mathrm{g} / \mathrm{g}$. Se hace notar que todas las estaciones de muestreo superaron los niveles para contaminación moderada indicados para este elemento 3 a $8 \mu \mathrm{g} / \mathrm{g}$, y que cinco estaciones superaron los niveles de contaminación alta 8 $\mu \mathrm{g} / \mathrm{g}$, lo que nos indicaria que toda la zona está en la categoría de contaminación alta según la USEPA (1991).

Según la OME de Canadá, todas las estaciones de muestreo están dentro del rango de sedimentos contaminados para arsénico que establece como límite 8 $\mu \mathrm{g} / \mathrm{g}$, para este metal, según los resultados obtenidos en la Bahía de Ite, la media obtenida se encuentra por arriba del este límite. Además en lo que respecta a los límites establecidos por la NOAA, para la concentración de un elemento que raramente produce efecto toxico, es de 8.2 $\mu \mathrm{g} / \mathrm{g}$.

\subsection{Cadmio}

La Figura 2d, muestra la evolución horizontal por isolineas del contenido de cadmio en los sedimentos superficiales de la Bahía de Ite. La concentración de este metal fluctúa entre $0.22 \mathrm{mg} / \mathrm{kg}$. (E-5) y $0.72 \mathrm{mg} / \mathrm{kg}$ (E-6) Tabla I, con una media de $0.41 \mathrm{mg} / \mathrm{kg}$ Tabla 1 . Como se puede observar estas concentraciones están por debajo de la concentración de $1.0 \mu \mathrm{g} / \mathrm{g}$ para sedimentos no contaminados (Sadiq 1992).

También notamos que, según los estándares para sedimentos marinos establecidos por la Agencia de Protección Ambiental de Estados Unidos (USEPA). para el metal pesado cadmio las concentraciones encontradas en la Bahia de Ite, están en la categoria de sedimentos no contaminados debido a que no superan los $6.0 \mu \mathrm{g} / \mathrm{g}$. Se hace notar que todas las estaciones de muestreo no superaron los niveles antes indicados para este elemento, lo que nos indicaría que toda la zona está en la categoria de no contaminada según la USEPA (1991).

De igual manera si efectuamos comparaciones con los niveles de concentración de metales en sedimentos por el Ministerio Ambiental de Ontario (OME, 1991) para zinc, que establece como limite $1.0 \mu \mathrm{g} / \mathrm{g}$, para este metal según los resultados obtenidos la Bahía de lte se encuentra muy por debajo de este límite, en promedio $0.41 \mathrm{mg} / \mathrm{kg}$. Además de estar bajo el rango de causa de efectos tóxicos, establecidos por la NOAA, que establece como umbral $1.2 \mu \mathrm{g} / \mathrm{g}$, concentración de un elemento que raramente produce efecto tóxico.

Realizada la comparación entre los resultados de las concentraciones de cadmio obtenidos en la Bahia de Ite, con una media de $0.41 \mathrm{mg} / \mathrm{kg}$ (Tabla 1), y los resultados que se obtuvo en la zona de Las Mesas con una media de $0.160 \mathrm{mg} / \mathrm{kg}$ notamos la diferencia existente a pesar que no supera los niveles críticos de contaminación, y los valores medios de cadmio encontrados en la zona norte de Santa Rosa con $0.336 \mathrm{mg} / \mathrm{kg}$. Estos resultados significan que la proyección de la contaminación tiene influencia en la zona de Santa Rosa.

\subsection{Mercurio}

Los resultados de las concentraciones de mercurio obtenidas en la Bahía de Ite, se muestran en la Tabla 2 valores que no superaron los niveles de cuantificación del laboratorio para sedimentos marinos, $<0.01 \mathrm{mg} / \mathrm{kg}$. De estos resultados podemos inferir que la presencia de mercurio en la zona está descartada, por efectos de la actividad minera y al efecto generado por los residuos de las minas de Toquepala y Cuajone que ha almacenado en las playas de la Bahía de Ite por más de 35 años.

\subsection{Plomo}

La Figura 2d muestra la distribución espacial por isolíneas de la concentración de plomo en los sedimentos superficiales de la Bahia de Ite, la cual fluctúa entre $6.18 \mathrm{mg} / \mathrm{kg}$ en la (E-1) y $10.50 \mathrm{mg} / \mathrm{kg}$ en la (E-9), con un valor medio de $8.472 \mathrm{mg} / \mathrm{kg}$ (Tabla I). Se puede apreciar las isolíneas de menor nivel de concentración de plomo se encuentran más cercanas al borde costero, extremos norte y sur, en la zona central las concentraciones son altas y conforme se alejan de la costa los niveles se incrementan progresivamente.

La mayor concentración de plomo total $(10.50 \mathrm{mg} / \mathrm{kg})$ se detectó en la E-9 (a $7.92 \mathrm{~km}$ de la boca del río Locumba) a una profundidad de $19.7 \mathrm{~m}$, esta concentración es inferior al rango $(19 \mathrm{a} 54 \mu \mathrm{g} / \mathrm{g}$ ) del sedimento superficial de la bahía San Vicente. Chile calificada de no contaminada (Ahumada, 1992). El menor valor $(6.18 \mathrm{mg} / \mathrm{kg}$ ) se registró en la E-1 (a 3.70 $\mathrm{km}$ de la boca del río Locumba) a una profundidad de $11.9 \mathrm{~m}$ tabla 1. En la evaluación la distribución espacial de plomo es creciente de la línea de costa cerca a la boca del río Locumba, que va incrementándose hacia afuera de la costa y preferentemente a los extremos e incidiendo más al noroeste frente a Santa Rosa, Sánchez, et al. 1995, efectuó evaluación de metales trazas en la zona, se reporto valores de plomo que fluctuaron de $8.00 \mathrm{a} 25.00 \mu \mathrm{g} / \mathrm{g}$ considerados como poco significativos.

Comparando los resultados obtenidos con los criterios de concentración de metales en sedimentos por el Ministerio Ambiental de Ontario (OME 1991), que establece como limite $50 \mu \mathrm{g} / \mathrm{g}$, para el plomo, según los resultados obtenidos la Bahía de Ite se encuentra muy por debajo de este límite. Además de estar bajo el rango de concentración de un elemento que taramente produce efectos tóxicos establecidos por la NOAA, que propone como umbral $46.7 \mu \mathrm{g} / \mathrm{g}$, todas las estaciones de muestreo se encuentran bajo este nivel de referencia.

Resultados reportados por CORDETACNA 1984, en sedimentos marinos para el plomo en la Bahía de Ite indican un nivel de $10.00 \mu \mathrm{g} / \mathrm{g}$. Balbin 1995, indica que para sedimentos marinos un nivel de plomo es de 8.73 $\mathrm{mg} / \mathrm{kg}$. PAMA SPCC 1994, a $10 \mathrm{~m}$ de profundidad reporta niveles promedios de plomo de $11.8 \mathrm{mg} / \mathrm{kg}$ en sedimentos marinos. 
Las concentraciones de plomo encontradas en los sedimentos superficiales de la Bahía de Ite, estarían indicando un nivel de degradación de la calidad de estos, aunque los niveles no superen los estándares establecidos por algunas entidades internacionales generan impacto negativo al ecosistema.

\subsection{Hierro}

La Figura 2e muestra como está distribuido horizontalmente el hierro en los sedimentos superficiales de la Bahía de Ite, el cual fluctúa entre los niveles de $14596.37 \mathrm{mg} / \mathrm{kg}$ en la (E-7) y 81106.98 $\mathrm{mg} / \mathrm{kg}$ en la (E-4) tabla 1, con un valor promedio de $33078.633 \mathrm{mg} / \mathrm{kg}$. La distribución horizontal muestra una similitud con otros metales en los cuales la mayor concentración se encuentra cercana a la desembocadura de los relaves que utilizó el cauce del río Locumba.

También podemos observar que, según los estándares para sedimentos marinos establecidos por la Agencia de Protección Ambiental de Estados Unidos (USEPA), para el metal pesado hierro las concentraciones encontradas en la Bahía de Ite, están en la categoría de sedimentos contaminados debido a que superan los $17000.00 \mu \mathrm{g} / \mathrm{g}$. Se hace notar que todas las estaciones de muestreo superaron los niveles antes indicados para este elemento, salvo la E-7, que tiene $14596.37 \mathrm{mg} / \mathrm{kg}$, lo que nos indicaría que toda la zona está en la categoría de contaminada según la USEPA (1991).

Todas las estaciones de muestreo están dentro del rango de sedimentos contaminados para hierro según la Guía de calidad sedimentos adoptado como referencia para el caso de lagos en Ontario, Canadá (Gonzales 2002), que establece un nivel para efecto leve de $2 \%$, y de $4 \%$ para un efecto grave. Según los resultados obtenidos la Bahia de Ite se encuentra con 2 estaciones que superan los niveles de efecto grave (E-4) y (E-6), y 6 estaciones que superan los niveles efecto leve, (E-1), (E-2), (E-3), (E5), (E-8) y (E-9).

Otras investigaciones realizadas reportan los siguientes resultados en la zona, Balbin 1995, indica que para sedimentos marinos un nivel de hierro de 39428.00 $\mathrm{mg} / \mathrm{kg}$. PAMA SPCC 1994, a $10 \mathrm{~m}$ de profundidad reporta niveles promedios de hierro de $3.63 \%$ en sedimentos marinos.

\section{CONCLUSIONES}

1. Los niveles de contaminación por metales ecotóxicos en los sedimentos marinos superficiales de la Bahía de Ite, provincia de Jorge Basadre Grohmann de Tacna, reportó valores superiores a los limites máximos permisibles, estipulados por la Environmental Protection Agency (EPA) en cobre $(608,063 \mathrm{mg} / \mathrm{kg}$ ), hierro (33 $078.63 \mathrm{mg} / \mathrm{kg}$ ) y arsénico $(8.66 \mathrm{mg} / \mathrm{kg}$ ); catalogando a la zona como contaminada por estos elementos. Los niveles de zinc $(39.923 \mathrm{mg} / \mathrm{kg})$, plomo $(8.472 \mathrm{mg} / \mathrm{kg})$ y cadmio $(0.41 \mathrm{mg} / \mathrm{kg})$ no superaron los límites máximos permisibles para zonas contaminadas establecidos por la EPA, en consecuencia la zona de estudio estaría catalogada como no contaminada por estos metales ecotóxicos.

2. Al efectuar la comparación de los resultados obtenidos en la Bahía de Ite, con los valores reportados en la zona sur de Las Mesas o Punta Mesa, libre de efectos antropogénicos, obtenemos diferencias significativas en las concentraciones en los metales ecotóxicos: $\mathrm{Fe}, \mathrm{Cu}, \mathrm{Zn}, \mathrm{As}, \mathrm{Pb}$ y Cd. Todos los metales a excepción del $\mathrm{Hg}$, tienen niveles superiores a Las Mesas, lo que significa que existe contaminación antropogénica en la zona. La proyección de la contaminación a la zona norte de Santa Rosa, desde la Bahía de Ite se produce en algunos de los elementos analizados como el cobre, zinc y menor proporción el arsénico.

\section{REFERENCIAS BIBLIOGRÁFICAS}

Ahumada, Ramón: Vargas B. José. 1992. Tasas de Sedimentación como ruta de enriquecimiento de metales en el sedimento de una Bahía. Facultad de Ciencias. Universidad Católica de la Santísima Concepción.. Concepción. Chille.

Aranda, S. 1999. Condiciones Geoquimicas de los Sedimentos superficiales de la Ensenada Grande del Obispo. Trab. de Grado de Lic. en Química, Universidad del Oriente, Cumana, Venezuela.

Arias de Díaz, Alicia; García, Jose; 2001. Concentración de los metales pesados $\mathrm{Cu}$ y $\mathrm{Pb}$ y su relación con la actividad enzimática glucógeno fosforilasa y glucógeno sintetasa en el mejillón (Perna viridis), Zootecnia Trop., 19(2): 115-129.

Balls, P., S. Hull, B. Miller, J. Pirie \&W. Proctor. 1997. Trace metal in Scottish estuarine and coastal sediments. Mar. Pol. Bull. 34(1): 42-50.

Bonilla, J. Fermin. B. Gamboa \& M Carrera. 1995. Aspectos Geoquímicos de los Sedimentos Superficiales del Ecosistema Marino Costero de Jose. Estado Anzoategui, Venezuela. Bol. Instituto Oceanográfico Venezuela. Univ. Oriente.

Bonilla, J. Aranda, S. Ramírez, C. Moya, J. Márquez A. 2003. Calidad de los Sedimentos Superficiales de la Ensenada Grande del Obispo, Estado Sucre Venezuela. Instituto Oceanográfico de Venezuela, Universidad del Oriente, Cumaná, Venezuela.

Codina, J. \& A. Pérez. 2001. Metales pesados como polucionantes tóxicos. Enviro. Contam. Toxicol. 25: 250-254.

Corporación Departamental de Desarrollo de Tacna, 1984. Estudio Contaminación de Recursos Hídricos e Hidrobiológicos - Tacna. Volumen II, Tacna Perú.

Durán, I.L., M.V. Fuentes \& J.A. Gómez H. 2004. Concentración de cadmio, plomo y cobre en Anadara tuberculosa del manglar de la Isla Taborcillo, Punta Chame, República de Panamá. Tecnociencia. Vol .6, No2,91-104. 
Garcia-Montelongo, F., C. Díaz., L. Galindo, M.S. Larrechi \& X. Rius. 1994. Heavy metals in three fish species from the coastal waters of Santa Cruz de Tenerife (Canary Islands). Scientia Marina 58 (3) 179 183.

Lewis, B. L. \& W. M. Landing. 1992. The investigation of dissolved and suspended particulate trace metal fractionation in the Black Sea. Mar. Chem. 40: 105-141.

Martinez, G \& W. Senior. 2002. Especiación de Cd, Zn. $\mathrm{Cr}$ y $\mathrm{Pb}$ en núcleos de sedimentos de la Bahía de Bergantin, Edo. Anzoátegui, Venezuela. Interciencia. Vol. 27 No $4,173-179$

Moreno, Claudia. 2001. Métodos para Medir la Biodiversidad. Oficina Regional de Ciencia y Tecnologia para América Latina y el Caribe, UNESCO. Zaragoza, España.

PNUMA: Diagnostico Regional sobre las Actividades y Fuentes Terrestres de Contaminación que Afectan los Ambientes Marino, Costero y Dulceacuicola Asociados en el Pacífico Sudeste. PNUMA / PAM Oficina de Coordinación y CPPS (1999). 73 pp.

Ponce, R., J. Forja \& A. Gómez. Parra. 2000. Influencia de la actividad antropogénica en la distribución vertical de $\mathrm{Zn}, \mathrm{Cd}, \mathrm{Pb}$ y $\mathrm{Cu}$ en agua intersticial y sedimentos marinos costeros (Bahia de Cádiz, SW de España). Ciencias Marinas 26(3): 479.502.

Sadiq, M. 1992. Toxic Metal Chemistry in Marine Environments. Marcel Dekker, Inc. New York, 389 pp.

Sánchez, Guadalupe: Enríquez, Edgardo; Morón, Octavio; Solis Juana; Chang, Flor. 1998. Características del Medio Marino, Fuentes y evaluación de la contaminación en la Bahía de Ilo, Costa de Ite a río Sama, Informe Progresivo, IMARPE, Lima.

Sánchez, G. y Zeballos J. 1988. Inventario de Fuentes de Contaminación Marina de origen terrestre; domésticos, industrials y mineros de Pisco. Ilo, Ite y Chimbote. Pimera parte. Procede del Inventario de Fuentes de Contaminación del Pacifico Sudeste. Documento CPPS/UCR, Plan de Acción Pacifico Sudeste con Apoyo del PNUMA, Bogota, Julio 1988.
SPCC, 1995. Programa de Adecuación y Manejo Ambiental (PAMA). Southern Peru Cooper Corporation. Mina de Toquepala.

USEPA. 1991. Criteria for sediment concentration of metals In: heavy metals contamination of sediments in the upper connecting channels of Greats Lakes, Hidrobiology 219, 307-315.

Urrutia T. Jorge, 1995. Estudio de la Contaminación por Relaves Mineros en la Bahia de Ite. Tesis para optar el Titulo de Ingeniero Pesquero, Oceanógrafo e Hidrobiólogo. Universidad Nacional Federico Villareal, Facultad de Oceanografía, Pesquería y Ciencias Alimentarias.

Williamson. R. y D. Motrisey. 2000. Stormwater contamination of urban estuaries. Predicting the build up of heavy metals in sediments. Estuaries 23 (1): 46-66.

WHO. 1995. Inorganic lead. Envirommental health criteria 165. World Health Organization http://www.inchem.org/documents/ehc/ehc/ehc165.ht $\mathrm{m}$.

WHO - WORLD HEALTH ORGANIZATION. Mercury - Environmental Aspects. Geneva: WHO. 1989. Environmental Health Criteria 86, 115p.

WHO - WORLD HEALTH ORGANIZATION. Inorganic Mercury. Geneva: WHO, 1991. Environmental Health Criteria 118, 168p.

\section{Correspondencia:}

Lorenzo Walter Ibárcena Fernández

Ciudad Universitaria Fundo "Los Granados"

Calle Miraflores s/n. Tacna. Perú.

wibarcena@gmail.com 